\title{
A MULTI-COMPONENT SYSTEM FOR DATA ACQUISITION AND VISUALIZATION IN THE GEOSCIENCES BASED ON UAVS, AUGMENTED AND VIRTUAL REALITY
}

\author{
S. Bernardes ${ }^{1}$,, M. Madden ${ }^{1}$, A. Knight ${ }^{1}$, N. Neel ${ }^{1}$, N. Morgan ${ }^{1}$, K. Cameron ${ }^{1}$, J. Knox ${ }^{2}$ \\ ${ }^{1}$ Center for Geospatial Research (CGR), Department of Geography, University of Georgia, Athens, Georgia, 30602, United States - \\ (sbernard, mmadden, drew.knight25, 13ncn, nicholas.morgan25, kevin.cameron25)@uga.edu \\ ${ }^{2}$ Department of Geography, University of Georgia, Athens, Georgia, 30602, United States - (johnknox)@uga.edu
}

Commission IV, WG IV/9

KEY WORDS: Unmanned aerial systems, virtual reality, augmented reality, mixed reality, geovisualization, education

\begin{abstract}
:
In this age of computer gaming, portable device video and high definition entertainment, students are exposed to sophisticated graphics and virtual reality every day. As a result, students arrive at universities with a high level of expectation and experience in visualization and 3D graphics. Traditional materials for education and outreach rely predominantly on two-dimensional displays of maps, photographs, data graphs/histograms and conceptual diagrams. Advances in geospatial technologies, including unmanned aerial systems and virtual/ augmented reality devices can be used to enhance and innovate instructional materials in classrooms from pre- $\mathrm{K}$ to graduate degree programs. This work reports on these technologies and the integration of the 3D Immersion and Geovisualization (3DIG) system at the Center for Geospatial Research at the University of Georgia, USA. We present system components, lessons learned during design and implementation of the system, and the incorporation of 3DIG into teaching, learning and research. Data flow is used as a multi-component system integrator and shows how interconnected and complementary technologies can provide hands-on and immersive experiential learning to students in the geosciences. System evaluation shows increased student interest/engagement and indicates that 3DIG facilitates the understanding of complex concepts.
\end{abstract}

\section{INTRODUCTION}

Students are increasingly visual learners and come to universities with a high level of expectation and experience in visualization strategies, including 3D graphics. Recent advances in technologies used by students, including a variety of mobilebased applications, computer gaming related hardware/software, and unmanned aerial systems, among others, can also be used to facilitate data acquisition and visualization in the geosciences. Although some of these technologies are progressively finding their way towards classrooms, materials for education and outreach in the geosciences rely predominantly on twodimensional displays of images, maps, photographs, data graphs and conceptual diagrams.

It is expected that the incorporation of recent technologies in traditional lectures and instructional materials will promote student learning and understanding of abstract and complex concepts. Further, these technologies should increase interest in geography, geology and many other areas dealing with the representation of the natural and man-made environments. Because of the use of cutting-edge technologies, increased interest may also be identified involving STEM related fields,

This paper summarizes the progress of efforts at the Center for Geospatial Research (CGR), Department of Geography, University of Georgia, United States, to bring together multiple technological components for cutting-edge data acquisition, virtual/augmented reality and geovisualization for enhanced Earth Science teaching and learning. With this project we aim to incorporate and test the proposed technologies in traditional lectures and instructional materials to facilitate student learning and understanding of hard to grasp concepts. The work assumes that the involved interconnected and complementary technologies can provide transformative hands-on and immersive learning experiences by exposing students in the geosciences to data acquisition, manipulation, visualization and analysis. Multiple components of our system invoke experiential learning and students have been actively involved in all phases of the project, including design, implementation and management of individual segments of the project. The sections below present the status of the 3D Immersion and Geovisualization (3DIG) system project, considering each major component of the data acquisition and visualization system, including: (1) Image Acquisition (UAS); (2) Virtual Reality; (3) Augmented/Mixed Reality; and (4) Video Wall. Sections and descriptions also emphasize system integration and data flow to illustrate system connectivity.

\section{THE 3D IMMERSION AND GEOVISUALIZATION (3DIG) SYSTEM}

We integrated a multi-component system for data acquisition and visualization using a variety of hardware platforms and commercial-off-the-shelf and free (sometimes also open source) software. Software and hardware received different degrees of augmentation and in-house customizations. 3DIG uses technologies that can be interconnected and that create opportunities for hands-on and immersive learning experiences spanning from system design to application. System design and implementation emphasized component integration and identified solutions that facilitate data flow between components. Major components of 3DIG include Image Acquisition, Virtual Reality, Augmented/Mixed Reality and 2D Panel (Video Wall). These components are presented and detailed in the following sections.

\footnotetext{
* Corresponding author
} 


\subsection{Image Acquisition Component}

3DIG currently incorporates three approaches for image acquisition: (a) Unmanned Aerial System (UAS), (b) mobile devices (tablets), and (c) 360-degree camera.

Image acquisition by UAS uses a customized DJI Phantom 4 Pro quadcopter, equipped with a 20 megapixel RGB camera. Features of the UAS, including attitude control, automatic takeoff \& landing, as well as collision avoidance, facilitate UAS flying by students with little or no previous exposure to this technology. Immersive First Person View (FPV) experience is available to users and employs a set of DJI Goggles featuring head tracking for drone control.

Enhancements to component design addressed a teaching/learning gap, as students had been taught remote sensing involving regions of the electromagnetic spectrum beyond visible, but had not had the opportunity to manipulate images they acquired in those spectral regions. This gap limited the ability of students to link field experience to remotelysensed products. The implemented solution incorporated a fiveband multispectral camera (MicaSense RedEdge) into the project. Spectral bands for this camera are presented in Table 1.

\begin{tabular}{|c|c|c|}
\hline Band Name & $\begin{array}{c}\text { Center } \\
\text { Wavelength } \\
(\mathbf{n m})\end{array}$ & $\begin{array}{c}\text { Bandwidth } \\
\text { FWHM } \\
(\mathbf{n m})\end{array}$ \\
\hline Blue & 475 & 20 \\
\hline Green & 560 & 20 \\
\hline Red & 668 & 10 \\
\hline Near IR & 840 & 40 \\
\hline Red Edge & 717 & 10 \\
\hline
\end{tabular}

Table 1. Multispectral band specifications for the MicaSense RedEdge camera (MicaSense, 2015)

A choice was made to keep the original high resolution RGB camera of the DJI Phantom 4 Pro when installing the MicaSense RedEdge, so users would have access to high spatial resolution RGB and lower spatial resolution multispectral, acquired simultaneously during a flight mission (Figure 1).

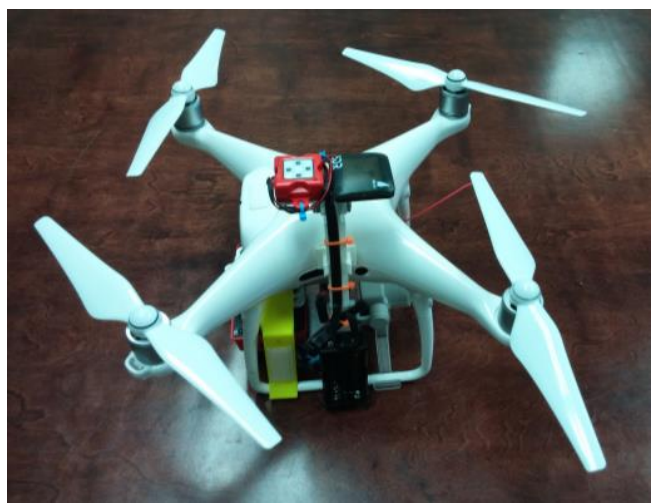

Figure 1. DJI Phantom 4 Pro quadcopter equipped with two cameras (RGB and five-band multispectral), and a mast supporting a GPS unit and downwelling radiation sensor

System integration required the design, 3D printing and testing of a mount for the MicaSense RedEdge that allows the full use of the Phantom's collision avoidance and downward vision systems. The integration of multispectral and RGB also exposes students to new requirements in data collection and field methods (e.g., rigorous radiometric calibration). Radiometric calibration is incorporated into the system by using a spectrally characterized calibrated reflectance panel. Data processing follows two data-dependent workflows involving radiometric calibration (multispectral only), structure-from-motion based point cloud generation, digital elevation model (DEM) and texture extraction, and the production of orthomosaics. Main applications of images acquired by the UAS include land cover mapping and 3D modeling.

Mobile devices (tablets) are used for flight planning, drone control during flights, and image acquisition monitoring in support of field campaigns. In addition, those devices have been used for image acquisition at ground level for project documentation, structure from motion and the creation of 360degree images/bubbles (see https://athenslayersoftime.uga.edu/). An alternative way of incorporating 360-degree images and video into the project involve a 360 -degree $5.7 \mathrm{~K}$ camera.

\subsection{Virtual Reality Component}

Immersive virtual reality is available in 3DIG through: (a) tethered virtual reality goggles, (b) mobile virtual reality headsets.

Tethered virtual reality goggles (Oculus Rift) have been used by graduate and undergraduate students members of our team to select game engines to produce detailed virtual reality environments. The project has explored the use of Unity and Unreal Engine platforms and has produced a series of 3D environments that are prepared for virtual reality. Figure 2 shows an example of one of these environments representing a section of the campus of the University of Georgia.

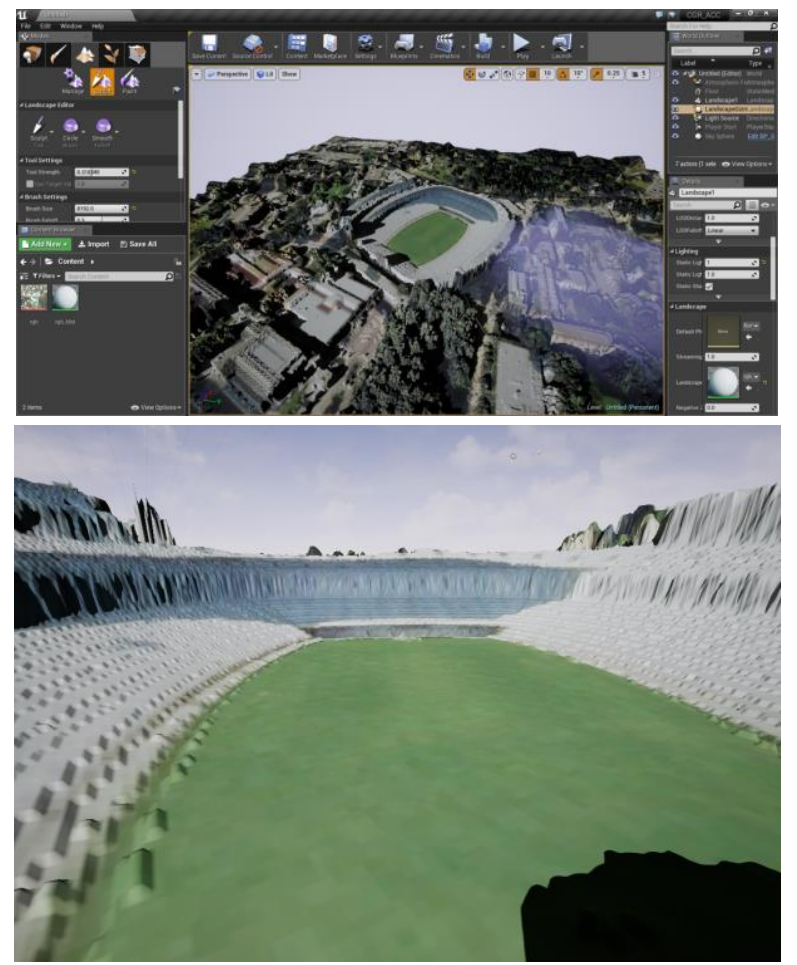

Figure 2. Virtual reality ready 3D environment representing the campus at the University of Georgia: Unreal Engine platform showing the Sanford Stadium (top), and a close view of the stadium (bottom) 
Multiple mobile virtual reality headsets are available for use in the classroom and to be borrowed by students. The headsets are compatible with iPhones and Androids and work with a variety of mobile phone sizes, so students can use their own application enabled phones to experience virtual reality, including video and audio.

\subsection{Augmented/Mixed Reality Component}

Immersive augmented/mixed reality capabilities are available through: (a) augmented reality sandbox, (b) mobile devices (iPad tablets), and (b) mixed reality headsets.

An augmented reality sandbox (Figure 3) was built in-house and allows for the near-real time augmentation of landforms created by users. Landforms are created by manipulating a variety of materials, including white sand and two types of sand enriched with a polymer to mimic the physical properties of wet sand. The digital sandbox system uses two Microsoft Xbox Kinect motion-sensing devices mounted over the sandbox and facing down. During operation, Kinect devices acquire distance (elevation) data at a high refresh rate and the data are transferred to a laptop computer for processing. Computations vary, depending on the application being used and concept being demonstrated. In its most basic form, an image representing elevation and contour lines are draped on the sand by using a short-throw projector. Software implementations currently being used with the sandbox include Augmented Reality Sandbox, developed by the University of California Davis (Augmented Reality Sandbox Development Team, 2018) and Tangible Landscape, developed by the GeoForAll Lab at North Carolina State University (Petrasova et al., 2018).

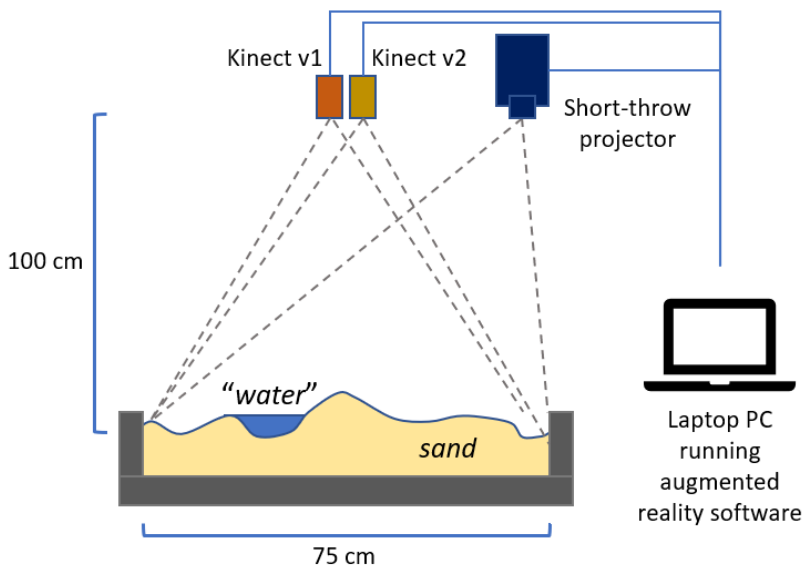

Figure 3. Components of the augmented reality sandbox

Multiple iPad tablets capable of running applications developed using Apple's augmented reality development platform (ARKit) are being incorporated into 3DIG. The tablets will be available to be borrowed by students and to be used with a variety of augmented reality applications in the classroom. Similarly, Microsoft HoloLens headsets are being incorporated into the project and allow for mixed reality experience.

\subsection{D Panel (Video Wall) Component}

The video wall is a low-maintenance and robust technical solution for 2D data visualization that includes four 46" highperformance commercial grade displays (NEC X464UNVTMX4P). The displays have thin bezel $(3.8 \mathrm{~mm})$ and are daisy chained for image tiling using TileMatrix technology. Assembling and maintenance is facilitated by the use of serviceable wall mounts and inter-display wall calibration. Video wall control is provided by an external computer (attached desktop or laptop) or a single Windows-based computer embedded into the first display.

\subsection{System Integration}

System integration was achieved by developing a data processing workflow that uses a combination of commercialoff-the shelf (COTS) tools and open source software. Preference was given to widely-used file formats, also taking into consideration file size and system performance, as files need to be transferred between components. We aimed at maximizing the contributions by each individual component of 3DIG and, during integration, we took advantage of existing documentation and explored/developed in-house solutions to fill gaps in knowledge and data flow. A diagram showing how the components of the system connect with each other is presented in Figure 4.

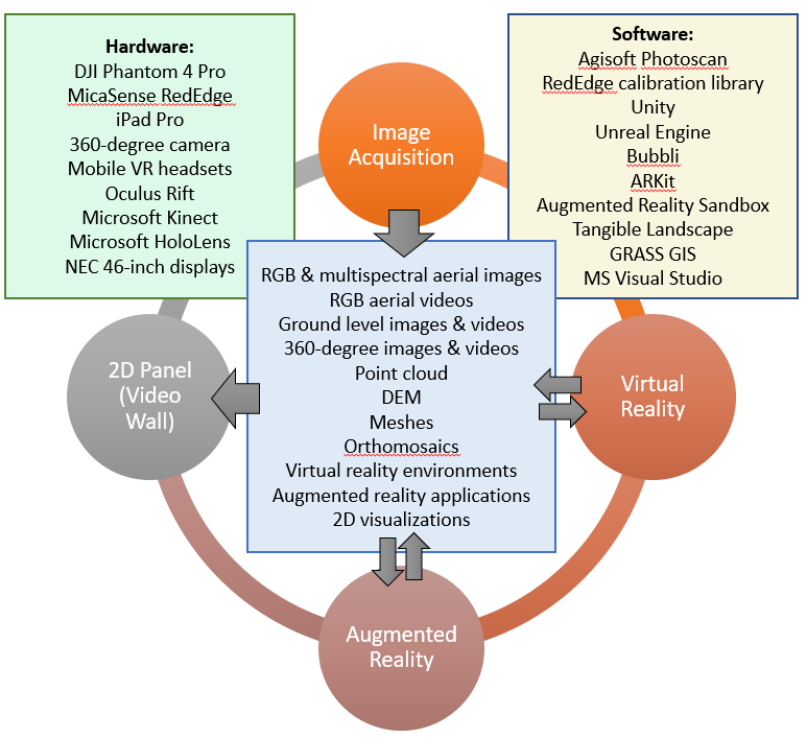

Figure 4. Components of the 3D Immersion and Geovisualization (3DIG) system and their integration

Seamless, near-real time integration is the goal of this project. In addition, due to the nature of the datasets being created and manipulated, solutions are being implemented considering the preservation of location, and particularly 3D attributes, during data flow. A summary of system connectivity includes the acquisition of multiple images by using the RGB and multispectral cameras mounted on the UAS. These data are processed using Agisoft Photoscan and structure from motion for 3D extraction. Results include point clouds, textured meshes, digital elevation models, and orthomosaics. A similar structure from motion procedure uses mobile tablets to acquired images on the ground for $3 \mathrm{D}$ reconstruction of surface objects. A 360-degree camera is used on the ground for image and video collection. Products derived from data collected by the UAS, tablets and 360-camera are then used with game engines (Unity and Unreal Engine) to support the creation of realistic models or 360-degree visualizations. Virtual reality headsets, including mobile headsets and Oculus Rift are then used for immersive visualization and interaction with data products. In addition, data products can be integrated into the augmented reality 
solution by draping images and models on the digital sandbox or by creating holographic representations of 3D models that can be scaled, rotated and explored by users. Use of augmented reality and data integration is also facilitated by the manipulation of layers in a GIS environment. Outputs can be further explored by using a variety of strategies for visualization of $2 \mathrm{D}$ and $3 \mathrm{D}$ datasets using the video wall.

\section{INCORPORATION INTO TEACHING, LEARNING AND RESEARCH}

\subsection{Strategies}

3DIG components have been used by students, faculty and staff at the university in classroom activities, presentations and research. In addition, the system has been used for community outreach and to facilitate demonstrations to visitors, including scholars and students from middle and high schools. System components invoke experiential learning and a team of graduate and undergraduate students at the Center for Geospatial Research at the University of Georgia has been actively involved in all phases of the 3DIG project, including design, implementation and management.

In particular, the augmented reality solutions, including the digital sandbox, have been used to illustrate multiple concepts in Geography and Geology, including landforms, hydrology and surface-atmosphere interactions. Sandbox users can easily modify topography (Figure 5) and responses to interaction are near-real time and can involve fluid flow over the landscape or running multi-layer based mathematical models (e.g., erosion model and fire model).

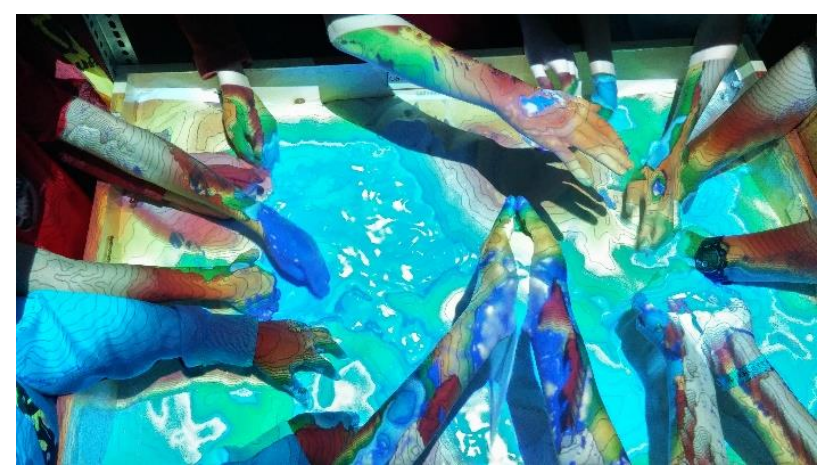

Figure 5. Students interact with the augmented reality sandbox and learn concepts in Geography and Geology

We estimate that more than 1500 students, faculty and staff have been exposed to the data collection and visualization capabilities of 3DIG over the first ten months of operation of the project. Courses that directly benefitted from 3DIG technologies include a variety of GIScience and remote sensing classes, physical geography, and weather and climate, taught at the Department of Geography at the university. Digital sandbox design requirements included the use of casters and a telescopic structure that holds sensors and projector. This structure can be lowered to fit through doors and elevators, facilitating the transport of the system to classrooms, laboratories and other buildings. Also because of that, 3DIG has provided opportunities to expose students taking courses outside Geography to technological advances in data acquisition, processing and visualization. These courses range from introductory to advanced level.
In addition to being used for teaching, components of 3DIG have been incorporated into research, including data collection, manipulation and analyses. In one research application, a team of students participating in the UGA's node of the NASA DEVELOP program has explored new material possibilities for the augmented reality sandbox and has worked on creative ways to produce realistic representations of topography in the Southern Appalachian Mountains region, United States. Results of this effort included the use of these realistic representations as input to fire models. Examples of other investigations involve the use of high resolution RGB, multispectral images and computer vision techniques for the detection of small targets in vegetated areas; for the study of plant traits and growth and to investigate vegetation disturbance following hurricanes.

\subsection{System Evaluation}

Effectiveness of 3DIG in facilitating the understanding of complex and hard-to-grasp concepts is being evaluated. We have worked with instructors on multiple courses on the development of material and applications to be presented using 3DIG. Instructors also assist in implementing an evaluation strategy based on surveys assessing knowledge before and after instructional material is presented using our system. We intend to compare statistics on grades achieved by students in key courses at the university before and after exposure to technologies. Over a longer time frame, we will track expected increases in Geography/Geology majors and enrolment in courses featuring the 3DIG over a 5 -year period.

Due to the novelty of the technologies involved, besides evaluating teaching/learning outcomes based only on grades, we also assess learning experience and student perception. 3DIG has an experimental nature and we want to assess student interest in learning more using similar strategies. Our surveys and observations show that the system increase engagement and promotes discussion and new approaches for problem solving. In particular, due to its characteristics, the digital sandbox has high potential for collaborative work and synergistic interactions when people from different backgrounds explore modelling possibilities. The modular nature of the system and the definition of procedures for component integration provide flexibility in system use.

Finally, 3DIG technologies lie at the cutting-edge of education/instruction. These technologies and their use are of interest at professional conferences in Geography, Geology. Education and others (Bernardes, Knight et al., 2017, Bernardes, Knight et al., 2018; Bernardes and Knight, 2017, Bernardes, Madden et al., 2017; Madden et al., 2018, Morgan et al., 2018 and Neel et al., 2017).

\section{CONCLUSIONS}

We took advantage of recent developments in a variety of fastpaced transformative industries (i.e., gaming, drone, mobile, virtual \& augmented reality, multimedia) and assembled and integrated a complex set of tools that can be used for data acquisition and visualization in the geosciences and other fields of knowledge. The tools support teaching/learning and research, and allow for communication of strategies for problem solving and exploration of what-if-scenarios.

Our assessments of system effectiveness in promoting student learning and motivation for learning (including how novel and 
interesting the technologies are to learners) indicate that the system fulfils student expectations regarding the incorporation of technologies in traditional lectures and instructional materials.

The 3DIG system continues to evolve and new technologies are added to the data acquisition and visualization toolsets, as they become available. Recent additions to 3DIG (HoloLens and ARKit enabled iPads) expand the mixed reality and sensor capabilities of the system. We are currently porting applications to be used on those platforms. Finally, further system expansion and access to technologies that complement the capabilities of 3DIG and that currently are not available in house (e.g., LiDAR) is being provided through collaborations with other groups.

\section{ACKNOWLEDGEMENTS}

The authors thank the Center for Teaching and Learning at the University of Georgia for the financial support.

\section{REFERENCES}

Augmented Reality Sandbox Development Team, 2018. Augmented Reality Sandbox Project. University of California Davis, W.M. Keck Center for Active Visualization in the Earth Sciences (KeckCAVES) https://arsandbox.ucdavis.edu/ (20 July 2018).

Bernardes, S., Knight, A., Neel, N., Weber, S., Ganguly, J., and Madden, M., 2018. Integration of UAVs, virtual and augmented reality into education and outreach: current status and lessons learned from the 3D Immersion and Geovisualization System (3DIG). Presentation at the Annual Conference of the American Society for Photogrammetry and Remote Sensing. Denver, CO.

Bernardes, S., Knight, A., Neel, N., Weber, S., and Madden, M., 2017. Incorporating emerging geospatial technologies into natural resource management at the Center for Geospatial Research, UGA. Presentation at the 11th Southern Forestry and Natural Resource Management GIS Conference. Athens, GA.

Bernardes, S. and Knight, A. 2017. Incorporating advanced geospatial technologies into education, research and outreach: The 3D Immersion and Geovisualization System (3DIG). Presentation at MapTime Athens. Athens, GA.

Bernardes, S., Madden, M., Knight, A., Cotten, D., and Jordan, T., 2017. Advanced geospatial technologies for education and outreach: 3D Immersion and Geovisualization system (3DIG). Presentation at the Annual Conference of the American Society for Photogrammetry and Remote Sensing. Baltimore, MD.

Madden, M., Bernardes, S., Cotten, D., and Jordan, T., 2018. Advanced geospatial technologies for agricultural landscapes: 3D Immersion and Geovisualization System (3DIG). Presentation at the US-IALE Annual Meeting. Chicago, IL.

MicaSense, 2015. MicaSense RedEdge 3 Multispectral Camera - User Manual, Revision 6, October 2015. 33 p.

Morgan, N., Knox, J., Bernardes, S., Cameron, K., Neel, N., and Knight A., 2018. Playing in the UGA sandbox: Incorporating Augmented Reality into Weather and Climate Classes. Presentation at the 98th Annual Meeting of the American Meteorological Society. Austin, TX.
Neel, N., Ganguly, J., Knight, A., and Bernardes, S., 2017. Creating an enhanced wildfire modeling tool using a tangible landscape system. Presentation at the 11th Southern Forestry and Natural Resource Management GIS Conference. Athens, GA.

Petrasova, A., Harmon, B., Petras, V., Tabrizian, P., Mitasova, H., 2018. Tangible modeling with open source GIS. Springer International Publishing, $202 \mathrm{p}$. 